\title{
THE INVARIANTS OF FORMS
} UNDER THE BINARY LINEAR HOMOGENEOUS GROUP $G_{6}$ MODULO $2 *$ BY O. E. GLENN

1. Introduction. The transformation of an arbitrary binary quantic whose coefficients are written without binomial multipliers,

$$
f=\left(a_{0}, \ldots, a_{m}\right)\left(x_{1}, x_{2}\right)^{m}=\prod_{i=0}^{m}\left(r_{2}^{(i)} x_{1}-r_{1}^{(i)} x_{2}\right),
$$

by the formulas

$$
T: x_{1}=\lambda_{1} x_{1}^{\prime}+\mu_{1} x_{2}^{\prime}, x_{2}=\lambda_{2} x_{1}^{\prime}+\mu_{2} x_{2}^{\prime},
$$

in which $\lambda_{i}, \mu j$ are such residues of a prime $p$ that $T$ ranges over the total group $G$ of order $\left(p^{2}-p\right)\left(p^{2}-1\right)$, leads to the formal modular concomitant system of $f$. For various reasons $p=2$ gives rise to exceptions in this theory; thus quadratic congruence theory becomes very special when $p=2$, and also certain types of modular concomitants exist $t$ for the even modulus that do not exist for $p>2 . \$$

2. Analogies. It is a known result of algebraic (nonmodular) invariant theory that every concomitant of (1) is a polynomial in determinants of two types, viz. $\left(r^{(i)} r^{(i)}\right),\left(r^{(i)} x\right)$, i. e., linear forms themselves and resultants of pairs of them. Also the complete system of covariants of any number of quadratic quantics,

(2) $f_{1}=\left(a_{0}, \ldots, a_{2}\right)\left(x_{1}, x_{2}\right)^{2}, \ldots, f_{r}=\left(l_{0}, \ldots, l_{2}\right)\left(x_{1}, x_{2}\right)^{2}$, is a set of concomitants that can be formed as transvectants of forms $f_{i}$ taken in pairs.\& The dyadic combinations therefore furnish the complete seminvariant systems, also, but for the invariants it is found that the eliminants of the triadic combinations of the forms $f_{1}, \ldots, f_{r}$ are to be added.

* Presented to the Society, December 28, 1923.

†Transactions of this Society, vol. 19 (1918), p. 110.

$\ddagger$ Dickson, The Madison Colloquium Lectures, Lecture III, p. 33-64.

$\S$ Grace and Young, Algebra of Invariants, 1903, p. 161. 
The facts are more complicated when the transformations form the group $G_{6}(\bmod 2)$. Then a simultaneous system of covariants of only two linear quantics

$$
f=a_{0} x_{1}+a_{1} x_{2}, \quad g=b_{0} x_{1}+b_{1} x_{2},
$$

is composed of sixteen concomitants. These are tabulated below. The methods for their derivation are exemplified later in this paper in the corresponding problem for two quadratics although the two problems are not without differences in respect to detail. We shall use the abbreviations

$$
\begin{aligned}
& E_{1}=a_{0}^{2} \frac{\partial}{\partial a_{0}}+a_{1}^{2} \frac{\partial}{\partial a_{1}}, \\
& E_{2}=b_{0}^{2} \frac{\partial}{\partial b_{0}}+b_{1}^{2} \frac{\partial}{\partial b_{1}}, \quad w=x_{1}^{2} \frac{\partial}{\partial x_{1}}+x_{2}^{2} \frac{\partial}{\partial x_{2}}
\end{aligned}
$$

and, if two linear covariants are

$$
C=c_{0} x_{1}+c_{1} x_{2}, D=d_{0} x_{1}+d_{1} x_{2},
$$

$[C D]$ is the covariant

$$
[C D]=\left(c_{0} d_{0}+c_{0} d_{1}+c_{1} d_{0}\right) x_{1}+\left(c_{1} d_{1}+c_{0} d_{1}+c_{1} d_{0}\right) x_{2} .
$$

The covariant $R$ in the list is led by the invariant (ab), viz.:

$$
\begin{aligned}
R=\left(a_{0} b_{1}\right. & \left.+a_{1} b_{0}\right) x_{1}^{3}+\left(a_{0} b_{1}+a_{0} b_{0}+a_{1} b_{1}\right) x_{1}^{2} x_{2} \\
& +\left(a_{1} b_{0}+a_{0} b_{0}+a_{1} b_{1}\right) x_{1} x_{2}^{2}+\left(a_{0} b_{1}+a_{1} b_{0}\right) x_{2}^{3} .
\end{aligned}
$$

Covariants of two linear forms under $G_{6}$ :

Invariants: $(a b), \quad I=a_{0}^{2}+a_{0} a_{1}+a_{1}^{2}, \quad J=b_{0}^{2}+b_{0} b_{1}+b_{1}^{2}$,

$$
L_{1}=a_{0}^{2} a_{1}+a_{0} a_{1}^{2}, \quad L_{2}=b_{0}^{2} b_{1}+b_{0} b_{1}^{2} .
$$

Linear Covariants: $f, g, E_{1} f, E_{2} g,[f g],\left[g E_{1} f\right]$,

$$
\left[f E_{2} G\right],\left[E_{1} f E_{2} g\right] \text {. }
$$

Quadratic covariants: $w f, w g, Q=x_{1}^{2}+x_{1} x_{2}+x_{2}^{2}$.

Cubic covariants: $R, L=x_{1}^{2} x_{2}+x_{1} x_{2}^{2}$.

Thus the independent covariants of a set of linear quantics, under $G_{6}$, form an extensive set which increases rapidly as the number of quantics is increased. If we add a third form $h=c_{0} x_{1}+c_{1} x_{2}$ to the set $f, g$ we shall have to consider concomitants formed from ground-forms in triadic combinations, as is proved by the existence of the following irreducible 
seminvariant:

$$
C=a_{0} b_{0} c_{1}+a_{0} b_{1} c_{1}+a_{1} b_{0} c_{1}+a_{1} b_{1} c_{0} .
$$

Similar considerations hold, under more complicated circumstances, for the simultaneous systems, for $G_{6}$, of a set of quadratic quantics. The rest of this paper concerns the system of two quadratics.

3. Seminvariants. Let $\boldsymbol{\Gamma}$ be the linear transformations upon $a_{0}, a_{1}, a_{2}$ which are induced by transforming $f$ by

$$
T: x=x_{1}^{\prime}+x_{2}^{\prime}, x_{2}=x_{2}^{\prime},
$$

and suppose $\Gamma^{\prime}$ to be that which corresponds by transformation of $g$ by $T$, where

'Then,

$$
f=a_{0} x_{1}^{2}+a_{1} x_{1} x_{2}+a_{2} x_{2}^{2}, g=b_{0} x_{1}^{2}+b_{1} x_{1} x_{2}+b_{2} x_{2}^{2} .
$$

$$
\begin{cases}\boldsymbol{\Gamma}: & a_{0}^{\prime}=a_{0}, \quad a_{1}^{\prime}=a_{1}, \quad a_{2}^{\prime}=a_{0}+a_{1}+a_{2} \\ \boldsymbol{\Gamma}^{\prime}: & b_{0}^{\prime}=b_{0}, \quad b_{1}^{\prime}=b_{1}, \quad b_{2}^{\prime}=b_{0}+b_{1}+b_{2} .\end{cases}
$$

With suitable restrictions we connect the problem of the seminvariants of $f$ and $g$ with a simpler problem, previously solved, by taking $a_{0}=0$, temporarily. We then have two simultaneous groups $(\bmod 2)$, viz.,

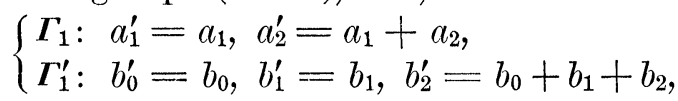

concerning which it is known that a fundamental system of universal concomitants consists of six quantics, as follows:*

$$
\begin{aligned}
a_{1}, b_{0}, b_{1}, \psi_{1}=a_{2}^{2}+a_{1} a_{2}, s= & \left(b_{0}+b_{1}+b_{2}\right) b_{2}, \\
& \varrho_{1}=a_{1} b_{2}+a_{2}\left(b_{0}+b_{1}\right) .
\end{aligned}
$$

We desire six seminvariants of the set $f, g$ such that the forms (10) are respectively residual to the six when $\alpha_{0} \equiv 0$ $(\bmod 2)$.

The seminvariant of $f$ of the type of $s$ is $a_{0} a_{2}+\psi_{1}=\sigma$. The leading coefficient of $[f g] \dagger$ is $a_{0} b_{0}+(f g)$, where $(f g)$ is the invariant

$$
(f g)=\left(a_{0}+a_{1}\right)\left(b_{1}+b_{2}\right)+\left(b_{0}+b_{1}\right)\left(a_{1}+a_{2}\right)+a_{1} b_{1} \text {. }
$$

The form $\varrho_{1}$ is the residue $\left(\bmod a_{0}\right)$ of $(f g)+\left(b_{0}+b_{1}\right) a_{1}=x$.

* Transactions of this Society, vol. 21 (1920), p. 293.

$\dagger$ The symbolism $(f g),[f g],\{f g\},\{\overline{f g}\}$, explained in (19) in the present article, was first defined in Procendings of the National. Academy, vol. 5 (1919), p. 107. 
Assume, in arbitrary form, a seminvariant $S$ of the set $f, g$,

$$
S=S\left(a_{0}, a_{1}, a_{2}, b_{0}, b_{1}, b_{2}\right)
$$

then, by (10),

$$
\begin{aligned}
S_{1}=S\left(0, a_{1}, a_{2}, b_{0}, b_{1}, b_{2}\right) & \\
& \equiv F\left(a_{1}, b_{0}, b_{1}, \psi_{1}, s, \varrho_{1}\right) \quad(\bmod 2),
\end{aligned}
$$

where $F$ is a polynomial in its arguments with integral coefficients. Hence we can arrange $S$ as an expression of the form

$$
\begin{aligned}
& S \equiv F\left(a_{1}, b_{0}, b_{1}, \sigma, s, x\right) \\
& +a_{0} F_{1}\left(a_{0}, a_{1}, a_{2}, b_{0}, b_{1}, b_{2}\right) \quad(\bmod 2),
\end{aligned}
$$

and $F_{1}$ is evidently a seminvariant of the set $f, g$. This process of reduction can be applied successively until we reach a coefficient quantic $F_{r}$ which is free from $a_{0}$ (explicitly), it being, therefore, a polynomial in

$$
a_{1}, b_{0}, b_{1}, \sigma, s, x
$$

$$
S \equiv F+a_{0} F_{1}+a_{0}^{2} F_{2}+\cdots+a_{0}^{r} F_{r} \quad(\bmod 2) .
$$

Theorem. A fundamental system of seminvariants of the set $f, g(\bmod 2)$ consists of the seven forms

$$
a_{0}, b_{0}, a_{1}, b_{1}, \sigma, s,(f g) \text {. }
$$

4. Syzygies. The expressions $k=a_{0} \sigma, \quad x=b_{0} s, a_{1}, b_{1}$, $q_{1}=\sigma+a_{0}^{2}+a_{0} a_{1}, q_{2}=s+b_{0}^{2}+b_{0} b_{1},(f g)$ are pure invariants. The following syzygies can be verified:

$$
\left\{\begin{array}{l}
a_{0}^{3}+a_{0}^{2} a_{1}+a_{0} q_{1}+k=0 \\
b_{0}^{3}+b_{0}^{2} b_{1}+b_{0} q_{2}+x=0 \\
\varrho^{2}+\left(a_{0}+a_{1}\right)\left(b_{0}+b_{1}\right) \varrho+\left(a_{0}^{2}+a_{1}^{2}\right) s+\left(b_{0}^{2}+b_{1}^{2}\right) \sigma \\
\quad+\left(a_{0} b_{0}+a_{1} b_{1}\right)\left(a_{0} b_{1}+a_{1} b_{0}\right)=0
\end{array}\right.
$$

where $\varrho=(f g)+a_{1} b_{1}$.

These syzygies may be employed as literal moduli of reduction for the purpose of reducing the arbitrary seminvariant $S\left(a_{0}, a_{1}, a_{2}, b_{0}, b_{1}, b_{2}\right)$ to a polynomial of finite order in $a_{0}, b_{0}$. We have immediately the following theorem.

THEOREM. The arbitrary seminvariant $S$ of two quadratic forms $f, g$ can be represented in the finite form

$$
\begin{aligned}
S=\Phi_{0}+\Phi_{1} b_{0}+\Phi_{2} b_{0}^{2}+a_{0}\left(\psi_{0}\right. & \left.+\psi_{1} b_{0}+\psi_{2} b_{0}^{2}\right) \\
& +a_{0}^{2}\left(\chi_{0}+\chi_{1} b_{0}+\chi_{2} b_{0}^{2}\right),
\end{aligned}
$$


in which $\boldsymbol{\Phi}_{i}, \psi_{i}, \chi_{i}$ are polynomials in the invariants

$$
q_{1}, q_{2}, k, \varkappa, a_{1}, b_{1},(f g),
$$

and the highest power of $(f g)$ which occurs is the first.

5. A Method in Covariants. It is known that a complete concomitant scale, ${ }^{*}$ for the modulus 2 , of a covariant,

$$
C=C_{0} x_{1}^{M}+C_{1} x_{1}^{M-1} x_{2}+\cdots+C_{M} x_{2}^{M},
$$

of a quantic $f_{m}$ of order $m$, for the reduction of all concomitants of degree unity in the coefficients of $C$ and of order $>3$, is composed of

$$
\left\{\begin{array}{c}
C,(C)=C_{1}+C_{2}+\cdots+C_{M-1} \\
{[C]=\left(C_{0}+(C)\right) x_{1}+\left((C)+C_{M}\right) x_{2}} \\
\{C\}=C_{0} x_{1}^{2}+(C) x_{1} x_{2}+C_{M} x_{2}^{2} \\
\{\bar{C}\}=C_{0} x_{1}^{3}+J_{1} x_{1}^{2} x_{2}+J_{2} x_{1} x_{2}^{2}+C_{M} x_{2}^{3} \\
\left(J_{1}+J_{2} \equiv(C)(\bmod 2)\right)
\end{array}\right.
$$

The latter covariant is existent only when $M$ is an odd number. This scale produces concomitants of $f_{m}$ from any covariant of the latter by the principle of copied forms.

Another method, not previously described, for the construction of covariants of $f_{m}$, to any prime modulus $p$, is to make an appropriate selection of a primary quantic,

$$
P_{0}=q_{0} x_{1}^{\alpha}+q_{1} x_{1}^{\alpha-1} x_{2}+\cdots+q_{\alpha} x_{2}^{\alpha},
$$

of given degree-order $(i, \alpha)$ and apply to it, simultaneously, the substitutions of the group upon the variables generated by $x_{1}=x_{1}^{\prime}+x_{2}^{\prime}, x_{2}=x_{2}^{\prime}$, and the corresponding substitutions of the induced group upon the coefficients. We thus obtain $p$ quantics

$$
\begin{aligned}
P_{0}, P_{1}=\left(q_{0}^{\prime}, \ldots, q_{\alpha}^{\prime}\right)\left(x_{1}, x_{2}\right)^{\alpha}, \ldots, \\
P_{p-1}=\left(q_{0}^{(p-1)}, \ldots, q_{\alpha}^{(p-1)}\right)\left(x_{1}, x_{2}\right)^{\alpha},
\end{aligned}
$$

and, if we assume that the primary quantic has been properly selected, any symmetric function of $P_{o}, P_{1}, \ldots, P_{p-1}$ is a covariant, modulo $p$, of $f_{m}$. Not many rules, other than em-

* Transactions of this Society, vol. 19 (1918), p. 110; vol. 20 (1919), p. 155. 
pirical ones, for the determination of primary quantics, are known to the writer, but examples of the method are shown in the next paragraph. Obviously $P_{0}$ should be such that the assumed symmetric function is invariantive under the other two generators of the total group $(\bmod p)$, i. e., $x_{1}=x_{1}^{\prime}, x_{2}=\lambda x_{2}^{\prime} ; x_{1}=x_{2}^{\prime}, x_{2}=-x_{1}^{\prime}$.

6. Fundamental Covariants. We are evidently able to reduce all covariants in terms of those of orders $0,1,2,3$ led by seminvariants $a_{0}^{i} b_{0}^{j},(i, j=0,1,2)$ and by the invariants of which $\Phi_{0}$ in (17) is a function. A formula showing this reduction in general form will be derived.

We find the following covariants with the leading coefficients which are adjoined. Abbreviations employed are

Linear forms:

$$
\begin{aligned}
& E_{1}=a_{0}^{2} \frac{\partial}{\partial a_{0}}+a_{1}^{2} \frac{\partial}{\partial a_{1}}+a_{2}^{2} \frac{\partial}{\partial a_{2}}, \\
& E_{2}=b_{0}^{2} \frac{\partial}{\partial b_{0}}+b_{1}^{2} \frac{\partial}{\partial b_{1}}+b_{2}^{2} \frac{\partial}{\partial b_{2}} .
\end{aligned}
$$

$$
\begin{aligned}
& a_{0}+a_{1},[f] ; b_{0}+b_{1},[g] ; a_{0} b_{0}+(f g),[f g] ; \\
& a_{0}^{2}+a_{1}^{2},\left[E_{1} f\right] ; b_{0}^{2}+b_{1}^{2},\left[E_{2} g\right] ; a_{0} b_{0}^{2}+\left(f E_{2} g\right),\left[f E_{2} g\right] ; \\
& a_{0}^{2} b_{0}+\left(g E_{1} f\right),\left[g E_{1} f\right] ; a_{0}^{2} b_{0}^{2}+\left(E_{1} f E_{2} g\right),\left[E_{1} f E_{2} g\right] .
\end{aligned}
$$

Quadratic forms:

$$
\begin{array}{lll}
a_{0}, f ; \quad b_{0}, g ; & a_{0}^{2}, E_{1} f ; \quad b_{0}^{2}, E_{2} g ; \quad a_{0} b_{0},\{f g\} ; \\
a_{0}^{2} b_{0},\left\{g E_{1} f\right\} ; & a_{0} b_{0}^{2},\left\{f E_{2} g\right\} ; \quad a_{0}^{2} b_{0}^{2},\left\{E_{1} f E_{2} g\right\} .
\end{array}
$$

There are no linear covariants, in the domain, led by invariants, and the only quadratic covariants whose leading coefficients are invariants are comprised in the formula $I Q$, where $I$ is an arbitrary invariant. The only invariantive leader of covariants of the third order which we shall be required to consider is $\Phi_{0}$ (cf. (17)). Let $\zeta$ be the operation of applying to a primary quantic $P_{0}$ the substitutions

If the primary is

$$
\begin{aligned}
& x_{1}=x_{1}^{\prime}+x_{2}^{\prime}, \quad x_{2}=x_{2}^{\prime} . \\
& \quad a_{0}^{\prime}=a_{0}, \quad a_{1}^{\prime}=a_{1}, \quad a_{2}^{\prime}=a_{0}+a_{1}+a_{2} .
\end{aligned}
$$

$$
P_{0}=\left(a_{0}+a_{2}\right) x_{1}+\left(a_{0}+a_{1}\right) x_{2},
$$

then

$$
P_{1}=\zeta P_{0}=\left(a_{1}+a_{2}\right) x_{1}+\left(a_{0}+a_{2}\right) x_{2},
$$


so that

$$
\left\{\begin{array}{l}
\sum P_{0}=\left(a_{0}+a_{1}\right) x_{1}+\left(a_{1}+a_{2}\right) x_{2}=[f] \\
\Delta_{1}=\sum P_{0} P_{1}=\left[\left(a_{0}+a_{2}\right) x_{1}+\left(a_{0}+a_{1}\right) x_{2}\right] \\
\quad \times\left[\left(a_{1}+a_{2}\right) x_{1}+\left(a_{0}+a_{2}\right) x_{2}\right] .
\end{array}\right.
$$

If the primary quantic is

$$
P_{0}=\left(b_{0}+b_{2}\right) x_{1}+\left(b_{0}+b_{1}\right) x_{2},
$$

we obtain, similarly,

$$
\left\{\begin{array}{l}
\sum P_{0}=\left(b_{0}+b_{1}\right) x_{1}+\left(b_{1}+b_{2}\right) x_{2}=[g] \\
\Delta_{2}=\sum P_{0} P_{1}=\left[\left(b_{0}+b_{2}\right) x_{1}+\left(b_{0}+b_{1}\right) x_{2}\right] \\
\quad \times\left[\left(b_{1}+b_{2}\right) x_{1}+\left(b_{0}+b_{2}\right) x_{2}\right]
\end{array}\right.
$$

The respective seminvariant leading coefficients of the covariants $[f] \Delta_{1},[g] \Delta_{2}$ are the invariants

$$
a_{1} q_{1}+k, \quad b_{1} q_{2}+\varkappa
$$

and these may replace $k, \varkappa$, respectively, in the system (18). Instead of $(f g)$ in the fundamental system (15), we may employ $(f g)_{1}=(f g)+a_{1} b_{1}$, which is the resultant of $[f]$ and $[g]$. A cubic covariant led by $(f g)_{1}$ is

$$
B=\left[f g \mid Q+[f] g+a_{1}[g] Q .\right.
$$

There exist no cubic covariants led by any of the invariants $k, x, q_{1}, q_{2}$, due to the fact that all of these invariants contain a term which is left unaltered by the permutational substitution* $\left(a_{0} a_{2}\right)\left(a_{1}\right)\left(b_{0} b_{2}\right)\left(b_{1}\right)=S_{1}$.

The cubic covariants which we require, with their leading coefficients, are listed below.

Cubic forms:

$a_{0}+a_{1},[f] Q ; \quad a_{0}^{2}+a_{1}^{2},\left[E_{1} f\right] Q ; \quad b_{0}+b_{1},[g] Q ;$

$b_{0}^{2}+b_{1}^{2},\left[E_{2} g\right] Q ; \quad a_{0} b_{0}+(f g),[f g] Q ; \quad a_{0}^{2} b_{0}+E_{1}(f g),\left[E_{1} f g\right] Q ;$ $a_{0} b_{0}^{2}+E_{2}(f g),\left[f E_{2} g\right] Q ; a_{0}^{2} b_{0}^{2}+E_{1} E_{2}(f g),\left[E_{1} f E_{2} g\right] Q ;(f g)_{1}, B$.

Theorem. An invariant leading coefficient $\boldsymbol{\Phi}_{0}$ of a cubic covariant of $f, g$ is necessarily congruent modulo 2 to the expression

$$
C=\left(a_{1} q_{1}+k\right) \psi_{1}^{\prime}+\left(b_{1} q_{2}+x\right) \psi_{2}^{\prime}+(f g)_{1} \psi_{3}^{\prime},
$$

where the quantics $\psi_{i}^{\prime}$ are invariants.

\footnotetext{
* Transactions of this Society, vol. 19 (1918), p. 111.
} 
To prove this we note that the invariant $\Phi_{0}$ (cf. (17)) is always of the form

$$
F\left(q_{1}, q_{2}, a_{1}, b_{1}\right)+C=\Phi_{0},
$$

where $F$ is an integral form in its arguments. We have constructed covariants whose leaders are the invariants of the set

$$
a_{1} q_{1}+k, b_{1} q_{2}+\varkappa,(f g)_{1} .
$$

If $G$ is a covariant whose leader is $\Phi_{0}$, we have that

$$
G+\psi_{1}^{\prime}[f] \Delta_{1}+\psi_{2}^{\prime}[g] \Delta_{2}+\psi_{3} B
$$

is a cubic covariant led by $F$, and this is an absurdity because every possible form $F$ evidently contains* a term which is left unaltered by $S_{1}$. Hence $F \equiv 0(\bmod 2), \Phi_{0} \equiv C$ (mod 2), which was to be proved.

7. Reductions of the Arbitrary Linear, Quadratic, and Cubic Covariants. Let $\Sigma_{1}$ represent a covariant of order unity whose seminvariant leading coefficient is $S$ (cf. 17)). We have, identically, $S=I+J$, where

$$
\left\{\begin{array}{l}
I=\left(b_{0}+b_{1}\right) \Phi_{1}+\left(b_{0}^{2}+b_{1}^{2}\right) \Phi_{2}+\left(a_{0}+a_{1}\right) \psi_{0} \\
\quad+\left[a_{0} b_{0}+(f g)\right] \psi_{1}+\left[a_{0} b_{0}^{2}+\left(f E_{2} g\right)\right] \psi_{2}+\left(a_{0}^{2}+a_{1}^{2}\right) \chi_{0} \\
\quad+\left[a_{0}^{2} b_{0}+\left(g E_{1} f\right)\right] \chi_{1}+\left[a_{0}^{2} b_{0}^{2}+\left(E_{1} f E_{2} g\right)\right] \chi_{2} \\
J=\Phi_{0}+b_{1} \Phi_{1}+b_{1}^{2} \Phi_{2}+a_{1} \psi_{0}+(f g) \psi_{1}+\left(f E_{2} g\right) \psi_{2} \\
\quad+a_{1}^{2} \chi_{0}+\left(g E_{1} f\right) \chi_{1}+\left(E_{1} f E_{2} g\right) \chi_{2} .
\end{array}\right.
$$

The following covariant is led by $I$ :

$$
\left\{\begin{array}{r}
K_{1}=\Phi_{1}[g]+\Phi_{2}\left[E_{2} g\right]+\psi_{0}[f]+\psi_{1}[f g]+\psi_{2}\left[f E_{2} g\right] \\
+\chi_{0}\left[E_{1} f\right]+\chi_{1}\left[g E_{1} f\right]+\chi_{2}\left[E_{1} f E_{2} G\right] .
\end{array}\right.
$$

Therefore, there would exist a linear covariant led by the invariant $J$, viz., $\Sigma_{1}+K_{1}$, unless $J \equiv 0(\bmod 2)$. Thus every linear covariant $\Sigma_{1}$ is reduced by the formula (32), $\left(\Sigma_{1}=K_{1}\right)$.

Let $\Sigma_{2}$ represent an arbitrary quadratic covariant which is led by $S$. The following covariant has $S$ for seminvariant leader:

$$
\left\{\begin{aligned}
K_{2}= & \Phi_{0} Q+\Phi_{1} g+\Phi_{2} E_{2} g+\psi_{0} f+\psi_{1}\{f g\} \\
& +\psi_{2}\left\{f E_{2} g\right\}+\chi_{0} E_{1} f+\chi_{1}\left\{g E_{1} f\right\}+\chi_{2}\left\{E_{1} f E_{2} g\right\}
\end{aligned}\right.
$$

Then $K_{2}$ differs from $\Sigma_{2}$ by some covariant which contains $x_{2}$

\footnotetext{
* The number $\left(\begin{array}{c}2 m \\ m\end{array}\right)$ is even for all integers $m$.
} 
as a factor; but, as no quadratic covariant can be factored thus, we have $\Sigma_{2} \equiv K_{2}(\bmod 2)$, i. e., the arbitrary quadratic covariant is reduced.

Let $\sum_{3}$ represent an arbitrary covariant of order 3 with seminvariant leader $S$. The following is a cubic covariant whose leading coefficient is $I$ (cf. (31)): $K_{3}=K_{1} Q$. Note therefore that $\Sigma_{3}+K_{3}=\Gamma_{3}$ is a covariant whose leader is $J$. Hence, by (28) (Theorem),

$$
J \equiv C+\left(f E_{2} g\right)_{1} \psi_{2}+\left(g E_{1} f\right)_{1} \chi_{1}+\left(E_{1} f E_{2} g\right)_{1} \chi_{2},
$$

that is,

$$
\begin{aligned}
\Gamma_{3}=\psi_{1}^{\prime}[f] \Delta_{1}+\psi_{2}^{\prime}[g] \Delta_{2}+\psi_{3}^{\prime} B & \\
& +\left(\psi_{2} E_{2}+\chi_{1} E_{1}+\chi_{2} E_{1} E_{2}\right) B,
\end{aligned}
$$

where $\left(f E_{2} g\right)_{1}=E_{2}(f g)_{1}=\left(f E_{2} g\right)+a_{1} b_{1}^{2}$, (cf. (27)). Note that operations by $E_{1}, E_{2}$ upon $B$ produce only polynomials in covariants already listed. We have now reduced $\Sigma_{3}$ to the form

$$
\sum_{3}=K_{3}+\Gamma_{3}+\Theta L \quad(\bmod 2),
$$

since $L$ is the only cubic covariant which contains $x_{2}$ as a factor. The quantic $\Theta$ is a pure invariant, but it is not known whether it is reducible, in all cases, entirely in terms of the invariants of the set (18). The following theorem has now been established.

Theorem. A fundamental system of formal covariants of the set consisting of the two binary quadratics

$$
f=\left(a_{0}, a_{1}, a_{2}\right)\left(x_{1}, x_{2}\right)^{2}, g=\left(b_{0}, b_{1}, b_{2}\right)\left(x_{1}, x_{2}\right)^{2},
$$

under the total group $G_{6}$, modulo 2 , is composed of 21 quantics, namely, seven invariants, $q_{1}, q_{2}, k, x, a_{1}, b_{1},(f g)$, eight linear covariants, $[f],[g],[f g],\left[E_{1} f\right],\left[E_{2} g\right],\left[f E_{2} g\right],\left[g E_{1} f\right],\left[E_{1} f E_{2} g\right]$, five quadratic covariants, $Q, f, g, \Delta_{1}, \Delta_{2}$, and one cubic covariant, $L$.

The remaining forms are reducible, as follows:

$$
\begin{gathered}
E_{1} f=[f]^{2}+a_{1}^{2} Q, \quad E_{2} g=[g]^{2}+b_{1}^{2} Q, \\
\{f g\}=[f][g]+a_{1} g+b_{1} f+a_{1} b_{1} Q, \quad\left\{g E_{1} f\right\}=E_{1}\{f g\}, \\
\left\{f E_{2} g\right\}=E_{2}\{f g\}, \quad\left\{E_{1} f E_{2} g\right\}=E_{1} E_{2}\{f g\} .
\end{gathered}
$$

The University of Pennsyluania 